HARDY-RAMANUJAN JOURNAL 36 (2013), 21-33

\title{
RAMANUJAN SERIES FOR ARITHMETICAL FUNCTIONS
}

\author{
M. RAM MURTY
}

\begin{abstract}
We give a short survey of old and new results in the theory of Ramanujan expansions for arithmetical functions.
\end{abstract}

\section{INTRODUCTION}

In 1918, Ramanujan [17] published a seminal paper entitled "On certain trigonometric sums and their applications in the theory of numbers" in which he introduced sums (now called Ramanujan sums) defined as

$$
c_{q}(n)=\sum_{\substack{a=1 \\(a, q)=1}}^{q} \cos \left(\frac{2 \pi a n}{q}\right)
$$

for any two natural numbers $q$ and $n$. It is easy to see that this can be re-written as

$$
c_{q}(n)=\sum_{\substack{a=1 \\(a, q)=1}}^{q} e^{2 \pi i a n / q}
$$

since $(a, q)=1$ if and only if $(q-a, q)=1$ so that we can pair up elements in (2) to derive (1).

These sums have remarkable properties. First, for fixed $n, c_{q}(n)$ is a multiplicative function. In other words, if $q_{1}, q_{2}$ are relatively prime, then

$$
c_{q_{1}}(n) c_{q_{2}}(n)=c_{q_{1} q_{2}}(n) .
$$

Second, from (2), it is easily seen that $c_{q}(n)$ is a periodic function of $n$ with period $q$. That is, the value of $c_{q}(n)$ depends only on the arithmetic progression of $n$ (mod $q)$. Third, using the familiar Möbius function, one can derive an explicit formula for $c_{q}(n)$ :

$$
c_{q}(n)=\sum_{d \mid(q, n)} \mu(q / d) d
$$

2010 Mathematics Subject Classification. Primary: 11-02, Secondary: 11A25.

Key words and phrases. Ramanujan sums, Ramanujan expansions, mean values, trigonometric series.

Research partially supported by a Simons Fellowship and an NSERC Discovery grant. 
where $\mu$ denotes the Möbius function. This is easily seen by using the characteristic property of the Möbius function:

$$
\sum_{d \mid n} \mu(d)=0, \quad n>1
$$

and is equal to 1 if $n=1$. Hence, from (2),

$$
c_{q}(n)=\sum_{a=1}^{q} e^{2 \pi i a n / q}\left(\sum_{d \mid(a, q)} \mu(d)\right)=\sum_{d \mid q} \mu(d)\left(\sum_{a=1, d \mid a}^{q} e^{2 \pi i a n / q}\right) .
$$

The inner sum can be re-written as

$$
\sum_{b=1}^{q / d} e^{2 \pi i b n /(q / d)}
$$

which equals $q / d$ if $n$ is divisible by $q / d$ and zero otherwise. Thus,

$$
c_{q}(n)=\sum_{d|q,(q / d)| n} \mu(d)(q / d)
$$

As $d$ runs over divisors of $q$ so does $q / d$ and we may re-write the above sum as

$$
c_{q}(n)=\sum_{d|q, d| n} \mu(q / d) d
$$

which is (3). In his work on the cyclotomic polynomial, Hölder [12] derived in 1936 the explicit formula:

$$
c_{q}(n)=\frac{\phi(q)}{\phi(q /(q, n))} \mu(q /(q, n)),
$$

where $\phi$ denotes Euler's function. More generally, one can study generalized Ramanujan sums of the form

$$
\sum_{d|q, d| n} f(d) g(q / d)
$$

with $f$ and $g$ arbitrary arithmetical functions, and derive some interesting results as in Apostol [1]. In a forthcoming paper, Fowler, Garcia and Karaali [4] show that many properties of Ramanujan sums can be deduced using the theory of supercharacters which seems to be an emerging new topic of group theory.

A convenient function to introduce is $\varepsilon_{d}(q)$ which is $d$ if $d \mid q$ and is zero otherwise. This allows us to write (3) as

$$
c_{q}(n)=\sum_{d \mid q} \varepsilon_{d}(n) \mu(q / d)
$$

so that by Möbius inversion we have

$$
\varepsilon_{q}(n)=\sum_{d \mid q} c_{d}(n) .
$$


This permits us to deduce several elegant Ramanujan expansions. For instance, let

$$
\sigma_{s}(n)=\sum_{d \mid n} d^{s}
$$

Then,

$$
\frac{\sigma_{s}(n)}{n^{s}}=\sum_{d \mid n} \frac{1}{d^{s}}=\sum_{d=1}^{\infty} \frac{1}{d^{s+1}} \varepsilon_{d}(n)=\sum_{d=1}^{\infty} \frac{1}{d^{s+1}} \sum_{q \mid d} c_{q}(n) .
$$

Interchanging summation, we find the sum is

$$
\frac{\sigma_{s}(n)}{n^{s}}=\zeta(s+1) \sum_{q=1}^{\infty} \frac{c_{q}(n)}{q^{s+1}}
$$

and all the sums are absolutely convergent for $\Re(s)>0$ since $\left|c_{q}(n)\right| \leq \sigma_{1}(n)$. This formula appears in [17].

One can derive several variations of this result. For instance, if we let

$$
\sigma_{s}(n, \chi)=\sum_{d \mid n} \chi(d) d^{s}
$$

then essentially the same argument as above shows that for any Dirichlet character $\chi(\bmod q)$, and $(n, q)=1$, we have

$$
\bar{\chi}(n) \frac{\sigma_{s}(n, \chi)}{n^{s}}=L(s+1, \chi) \sum_{q=1}^{\infty} \frac{\chi(q) c_{q}(n)}{q^{s+1}} .
$$

More elaborate examples of this nature can be found in [2]. Ivic [13] has shown that generally, for any completely multiplicative function $f$, we have by essentially the same argument that

$$
\sum_{d \mid n} f(d) d^{-s}=\left(\sum_{q=1}^{\infty} \frac{f(q)}{q^{s+1}}\right)\left(\sum_{q=1}^{\infty} \frac{f(q) c_{q}(n)}{n^{s+1}}\right) .
$$

Formula (3) also allows us to deduce that $c_{q}(n)$ is an ordinary integer though this is not obvious from the definition of $(1)$ or $(2)$. (One can also deduce this by viewing $(2)$ as the trace of the algebraic integer $\zeta_{q}^{n}$ in the cyclotomic field $\mathbb{Q}\left(\zeta_{q}\right)$.) Thus, if $(q, n)=1$, then $c_{q}(n)=\mu(q)$. Many of the basic properties of the Ramanujan sums are collected in [9].

In his paper, Ramanujan [17] derives a variety of expressions of the form

$$
\sum_{q=1}^{\infty} a_{q} c_{q}(n)
$$


for some arithmetical functions. For instance, for the divisor function, Ramanujan showed that

$$
d(n)=-\sum_{q=1}^{\infty} \frac{\log q}{q} c_{q}(n) .
$$

If $r(n)$ denotes the number of ways of writing $n$ as a sum of two squares, Ramanujan proves that

$$
r(n)=\pi \sum_{q=1}^{\infty} \frac{(-1)^{q}}{2 q+1} c_{2 q+1}(n) .
$$

We call series of the form (5) Ramanujan series (or Ramanujan expansion or sometimes Ramanujan-Fourier series) since such series mimic the notion of a Fourier expansion of an $L^{1}$-function. More precisely, given an arithmetical function $f$, we say that $f$ admits a Ramanujan expansion if

$$
f(n)=\sum_{q=1}^{\infty} \widehat{f}(q) c_{q}(n)
$$

for appropriate complex numbers $\widehat{f}(q)$ and the series on the right hand side converges. We say that $\widehat{f}(q)$ is the $q$-th Ramanujan coefficient of $f$.

Several natural questions now arise. First, for which arithmetical functions do we have such a series. If such a series exists for a given function $f$, then how can we determine the Ramanujan coefficients $\widehat{f}(q)$. What can we say about the rate of convergence of such a series? These are the questions we explore in this (largely survey) paper.

Some of these questions can be quite subtle. As Ramanujan observes in his paper [17], the assertion

$$
\sum_{q=1}^{\infty} \frac{c_{q}(n)}{q}=0
$$

is equivalent to the prime number theorem. This equation also shows that the Ramanujan coefficients of a given function need not be unique (since the above is an expansion of the zero function).

A more complicated example was given by Hardy [7] for the von Mangold function $\Lambda(n)$ defined as $\log p$ when $n$ is a power of the prime $p$ and zero otherwise:

$$
\frac{\phi(n)}{n} \Lambda(n)=\sum_{q=1}^{\infty} \frac{\mu(q)}{\phi(q)} c_{q}(n) .
$$

This expression, in conjunction with a Wiener-Khinchine type conjecture for Ramanujan expansions, was applied by Gadiyar and Padma [5] to give a heuristic derivation of the celebrated conjectural formula of Hardy and Littlewood on the 
number of twin primes up to $x$. We will describe their work below and take this opportunity to formulate a general question of Wiener-Khinchine type for a wide class of arithmetic functions. In a forthcoming paper, we plan to explore this in greater detail.

Regarding the first question of when such series expansions exist, there are a number of results for which we refer the reader to [18]. For instance, using functional analysis, Spilker [21] has proved that every bounded arithmetical function admits a Ramanujan expansion. Hildebrand [10] gave a surprisingly simpler proof of this result without the boundedness assumption. However, the Ramanujan coefficients in many of these cases are not "natural."

\section{An ORTHOGONALITY PRINCIPLE}

It was Carmichael [2] who noticed an orthogonality principle for the Ramanujan sums. This observation led him to predict what the Ramanujan coefficients of a given arithmetical function should be, if such an expansion exists. Indeed, given an arithmetical function $f$, we write $M(f)$ for the limit

$$
\lim _{x \rightarrow \infty} \frac{1}{x} \sum_{n \leq x} f(n)
$$

when it exists and call it the mean value of $f$. We also write $T_{h}$ for the shift operator: $T_{h}(f)(n)=f(n+h)$. Then,

Theorem 1. (Orthogonality relations)

$$
\lim _{x \rightarrow \infty} \frac{1}{x} \sum_{n \leq x} c_{r}(n) c_{s}(n)=\phi(r)
$$

if $r=s$ and zero otherwise. More generally,

$$
\lim _{x \rightarrow \infty} \frac{1}{x} \sum_{n \leq x} c_{r}(n) c_{s}(n+h)=c_{r}(h)
$$

if $r=s$ and zero otherwise. In other words, $M\left(c_{r} T_{h}\left(c_{s}\right)\right)=c_{r}(h)$ if $r=s$ and zero otherwise.

Proof. We evaluate

$$
\sum_{n \leq x} c_{r}(n) c_{s}(n)=\sum_{(a, r)=1} \sum_{(b, s)=1} \sum_{n \leq x} e^{2 \pi i(n(a / r+b / s))}
$$

Now, if $r \neq s$ then $a / r+b / s$ is not an integer. Indeed, if it were equal to $m$ (say), then

$$
a s+b r=m r s=b r+a s,
$$

from which we see that $r \mid s$ and $s \mid r$ so that $r=s$, a contradiction. Thus, if $r \neq s$, the innermost sum is a geometric sum which is bounded. Hence the limit in question 
is zero. If $r=s$, then the innermost sum is again bounded if $(a+b) / r$ is not an integer. Thus, the limit is non-zero only in the case $a \equiv-b(\bmod r)$ and the result is now immediate. For the second assertion, we proceed as before. Namely, we write out the Ramanujan sums. Interchanging summations, we see the limit in question is

$$
\sum_{\substack{b=1 \\(b, r)=1}}^{r} e^{2 \pi i h b / r} \sum_{\substack{a=1 \\(a, q)=1}}^{q} \lim _{x \rightarrow \infty} \frac{1}{x} \sum_{n \leq x} e^{2 \pi i n(a / q+b / r)} .
$$

As before, if $r \neq q$, then $a / q+b / r$ is not an integer and the innermost sum is bounded so that the limit is zero. If $r=q$, then the innermost sum is bounded unless $a+b \equiv 0$ $(\bmod q)$, in which case the limit is 1 . This completes the proof.

This orthogonality principle allows one to (heuristically) write down possible candidates for the Ramanujan coefficients of any given arithmetical function. Indeed, if

$$
f(n)=\sum_{q=1}^{\infty} \widehat{f}(q) c_{q}(n)
$$

then, multiplying both sides of the equation by $c_{r}(n)$ and taking the mean value of both sides, we find on interchanging the sum that

$$
\widehat{f}(r) \phi(r)=M\left(f c_{r}\right) .
$$

The second property allows us to deduce that if $f(n)$ admits a Ramanujan expansion with Ramanujan coefficients $\widehat{f}(q)$, then, so does the function $f(n+h)$ which has Ramanujan coefficients $\widehat{f}(q) c_{q}(h) / \phi(q)$.

There has been extensive study of when Ramanujan expansions exist and we refer the reader to some excellent surveys like [15] and [18] for details and additional references. However, in the next section, we highlight two important theorems due to Wintner and Delange (see for example, Cor. 2.3 of [18] or [23]) in this context. Generalizations of this theory to functions of several variables have been investigated by several authors, most notably, Ushiroya [22], but the theory is still in its infancy. For instance, one can investigate Ramanujan expansions of arithmetical functions of several variables and see under what conditions these expansions are valid.

\section{The theorems of Wintner And Delange}

The theorem of Wintner discussed below allows us to determine a large number of Ramanujan expansions.

Theorem 2. (Wintner, 1943) Suppose that

$$
f(n)=\sum_{d \mid n} g(d)
$$


and that

$$
\sum_{n=1}^{\infty} \frac{|g(n)|}{n}<\infty
$$

Then, $M(f)=\sum_{n=1}^{\infty} g(n) / n$.

Proof. We have

$$
\frac{1}{x} \sum_{n \leq x} f(n)=\frac{1}{x} \sum_{d \leq x} g(d)[x / d]=\sum_{d=1}^{\infty} \frac{g(d)}{d}+O\left(\sum_{d>x} \frac{|g(d)|}{d}\right)+O\left(\frac{1}{x} \sum_{d \leq x}|g(d)|\right) .
$$

By our hypothesis, the first big $O$-term goes to zero as $x$ tends to infinity. To study the last term, we need only apply partial summation. Let $S(x)=\sum_{n \leq x}|g(n)| / n$. Then,

$$
\sum_{n \leq x}|g(n)|=S(x) x-\int_{1}^{x} S(t) d t=\int_{1}^{x}(S(x)-S(t)) d t .
$$

Now fix $\epsilon>0$. Then, there is an $x_{0}$ such that $|S(x)-S(t)| \leq \epsilon$ for $x>t>x_{0}$. Thus, splitting the integral into two parts, we see that our sum in question is

$$
\leq 2 \alpha x_{0}+\epsilon x
$$

which completes the proof.

More generally, Wintner showed that if

$$
\sum_{n=1}^{\infty} \frac{|g(n)|}{n}<\infty
$$

then the Ramanujan coefficients $M\left(f c_{r}\right)$ exist for every $r$. If in addition,

$$
\sum_{n=1}^{\infty} \frac{|g(n)| d(n)}{n}<\infty
$$

then the Ramanujan expansion converges pointwise to $f(n)$. This was later improved by Delange [3] who proved the following theorem regarding Ramanujan expansions.

Theorem 3. (Delange, 1976) Suppose that

$$
f(n)=\sum_{d \mid n} g(d)
$$

and that

$$
\sum_{n=1}^{\infty} 2^{\omega(n)} \frac{|g(n)|}{n}<\infty,
$$

where $\omega(n)$ is the number of distinct prime divisors of $n$. Then, $f$ admits a Ramanujan expansion with

$$
\widehat{f}(q)=\sum_{m=1}^{\infty} \frac{g(q m)}{q m}
$$


The proof of Delange's theorem is not difficult and we refer the reader to [3] for complete details. However, we can here indicate the main ideas of the proof. One first shows that the double series

$$
\sum_{m, q \geq 1} \frac{g(q m)}{q m} c_{q}(n)
$$

is absolutely convergent. Once this is done, we evaluate

$$
\sum_{k=1}^{\infty} w_{k}
$$

where

$$
w_{k}=\sum_{q m=k} \frac{g(q m)}{q m} c_{q}(n)=\frac{g(k)}{k} \sum_{q \mid k} c_{q}(n) .
$$

As noted earlier, the latter sum is $\varepsilon_{k}(n)$ which is $k$ if $k \mid n$ and zero otherwise. Thus, $w_{k}=g(k)$ if $k \mid n$ and zero otherwise, from which the theorem follows immediately.

It is clear that the arithmetical function for $\Re(s)>0, \sigma_{s}(n) / n^{s}$ satisfies the conditions of Delange's theorem and thus admits a Ramanujan expansion and the Ramanujan coefficients are also determined from the theorem. Despite its simplicity and beauty, this theorem does not include the more subtle Ramanujan expansions of functions such as the divisor function or the von Mangoldt function. Several authors have developed larger theories that would enable one to derive such expansions. We refer the reader to the recent paper of Lucht [15] for details and additional references. For instance, Lucht proves:

Theorem 4. Let $\widehat{f}: \mathbb{N} \rightarrow \mathbb{C}$. If the series

$$
g(d):=d \sum_{m=1}^{\infty} \mu(m) \widehat{f}(d m)
$$

converges for every natural number $d$, then for

$$
f(n)=\sum_{d \mid n} g(d)
$$

we have

$$
f(n)=\sum_{q=1}^{\infty} \widehat{f}(q) c_{q}(n)
$$

This theorem allows one to deduce, for example, Ramanujan's expansion of the divisor function. Indeed, if we take

$$
\widehat{f}(n)=\frac{\log n}{n}
$$


then

$$
g(d)=\sum_{m=1}^{\infty} \frac{\mu(m)}{d m} \log d m=-1
$$

since

$$
\sum_{m=1}^{\infty} \frac{\mu(m)}{m}=0
$$

and

$$
\sum_{m=1}^{\infty} \frac{\mu(m)}{m} \log m=-1
$$

both assertions being quite subtle and requiring the theory of the Riemann zeta function. Indeed, the former is known to be equivalent to the prime number theorem. One can also deduce the Wintner-Delange theorems from this result. However, Hardy's Ramanujan expansion of $\phi(n) \Lambda(n) / n$ noted in the introduction, cannot be deduced from this result. Indeed, taking the cue from Hardy's expansion, we set

$$
g(d)=d \sum_{m=1}^{\infty} \frac{\mu(d m)}{\phi(d m)} \mu(m) .
$$

This series needs to converge for us to be able to deduce the Hardy expansion. Unfortunately, the series diverges since we may restrict the sum to those $m$ which are coprime to $d$ (since the Möbius function vanishes otherwise), in which case the summand is

$$
\mu(d) \mu^{2}(m) / \phi(d) \phi(m)
$$

and the series clearly diverges.

Hardy [7] obtains his expansion by considering the Dirichlet series

$$
f(s)=\sum_{q=1}^{\infty} \frac{\mu(q) c_{q}(n)}{q^{s-1} \phi(q)}
$$

which converges absolutely for $\Re(s)>1$. By the mutliplicative properties of the Ramanujan sum and the Euler function, we see that

$$
f(s)=\prod_{p}\left(1-\frac{c_{p}(n)}{p^{s-1} \phi(p)}\right),
$$

where the product is over all primes $p$. Now $c_{p}(n)=-1$ if $p$ is coprime to $n$ and $p-1$ otherwise. Thus,

$$
f(s)=\prod_{p \mid n}\left(1-\frac{1}{p^{s-1}}\right) \prod_{(p, n)=1}\left(1+\frac{1}{p^{s-1}(p-1)}\right) .
$$


We can rewrite this as

$$
\prod_{p \mid n}\left(\frac{(p-1)\left(p^{s-1}-1\right)}{p^{s}-p^{s-1}+1}\right) \prod_{p}\left(1+\frac{1}{p^{s-1}(p-1)}\right)=g(s) h(s) \quad \text { say. }
$$

It is evident that if $n$ is a prime power, $g(s)$ has a simple zero at $s=1$, and if $n$ is not a prime power, it has a zero of order at least 2 . On the other hand, it is not difficult to see that

$$
h(s)=\zeta(s) h_{1}(s)
$$

where $h_{1}(s)$ is regular for $\Re(s)>0$ and non-vanishing there. Thus, $f(s)$ is zero at $s=1$ when $n$ is not a prime power and is equal to

$$
\frac{p-1}{p} \log p
$$

if $n$ is a prime power. To complete the proof, we need to establish that the series converges to $f(1)$. But this can be deduced in several ways. Hardy deduces it by appealing to his earlier work with Littlewood [8] (see in particular Theorem D on page 218) where he showed that the prime number theorem is equivalent to the assertion that

$$
\sum_{n=1}^{\infty} \frac{\mu(n)}{n}=0
$$

A more direct method is to use Perron's formula for the partial sums and use contour integration which is a standard method in analytic number theory. The reader can find details of this method in [16].

\section{The Wiener-Khintchine Formula And AnAlogues}

In the theory of Fourier series, the Wiener-Khintchine formula states that if

$$
f(t)=\sum_{n} f_{n} e^{i \lambda_{n} t}
$$

then

$$
\lim _{T \rightarrow \infty} \frac{1}{2 T} \int_{-T}^{T} f(t+u) \overline{f(t)} d t=\sum_{n}\left|f_{n}\right|^{2} e^{i \lambda_{n} u} .
$$

Inspired by this theorem, Gadiyar and Padma [5] have asked if it is reasonable to expect that for a function $f(n)$ with Ramanujan coefficients $\widehat{f}(q)$, a similar result holds. Namely, is it true that

$$
\lim _{x \rightarrow \infty} \frac{1}{x} \sum_{n \leq x} f(n) f(n+h)=\sum_{q=1}^{\infty} \widehat{f}(q)^{2} c_{q}(h) ?
$$


We propose here a more general question. Given two arithmetical functions $f$ and $g$, when is it true that

$$
\lim _{x \rightarrow \infty} \frac{1}{x} \sum_{n \leq x} f(n) g(n+h)=\sum_{q=1}^{\infty} \widehat{f}(q) \overline{\widehat{g}(q)} c_{q}(h) .
$$

In a forthcoming paper [6], we show this is true for a wide class of functions. However, one must exercise some caution since there are already some contradictions, even in the simplest cases.

Still, the question is intriguing since as noted in [5], the case $f(n)=g(n)=$ $\phi(n) \Lambda(n) / n$ gives the Hardy-Littlewood prime-tuplet conjecture (which incidentally, was conjectured using the more sophisticated circle method). Indeed, from Hardy's expansion, we would have

$$
\lim _{x \rightarrow \infty} \frac{1}{x} \sum_{n \leq x} \frac{\phi(n)}{n} \frac{\phi(n+h)}{n+h} \Lambda(n) \Lambda(n+h)=\sum_{q=1}^{\infty} \frac{\mu^{2}(q)}{\phi^{2}(q)} c_{q}(h) .
$$

The last sum can be expressed as an infinite product over prime numbers:

$$
\prod_{p}\left(1+\frac{c_{p}(h)}{(p-1)^{2}}\right) \text {. }
$$

Since $c_{p}(h)=-1$ if $p$ is coprime to $h$ and $p-1$ if $p \mid h$, we see that this product is

$$
\prod_{p \mid h}\left(1+\frac{1}{p-1}\right) \prod_{(p, h)=1}\left(1-\frac{1}{(p-1)^{2}}\right)
$$

which agrees with the Hardy-Littlewood constant obtained by them using heuristic reasoning based on the circle method.

The conjecture can be viewed as a special case of a Parseval type formula in a suitable Hilbert space. Indeed, the authors in [11] consider the $\mathbb{C}$-linear span of all Ramanujan sums and consider the closure of this space (denoted $\mathcal{B}^{2}$ ) with respect to the (semi)-norm

$$
\|f\|_{2}^{2}:=\limsup _{x \rightarrow \infty} \frac{1}{x} \sum_{n \leq x}|f(n)|^{2} .
$$

For functions in this space, they establish using elementary Hilbert space theory, the Parseval identity:

$$
\|f\|_{2}^{2}=\sum_{q=1}^{\infty}|\widehat{f}(q)|^{2} \phi(q) .
$$

Indeed, the space $\mathcal{B}^{2}$ (modulo null functions) can be equipped with an inner product

$$
(f, g):=M(f \bar{g}) \text {. }
$$

This makes $\mathcal{B}^{2}$ into a Hilbert space and we have the general Parseval theorem from which our result above follows. This is not particular to our situation but a general 
fact about Hilbert spaces that the more specialized version of Parseval's formula implies the general one via a useful identity, namely,

$$
(f, g)=\frac{1}{4}\left(\|f+g\|_{2}^{2}-\|f-g\|_{2}^{2}+i\|f+i g\|_{2}^{2}-i\|f-i g\|_{2}^{2}\right) .
$$

The difficulty in extending this theorem to a wider class is formidable since as we said above, such a general theorem would lead to a proof of the twin-prime conjecture. Nevertheless, the theory of Ramanujan expansions does give a viable framework to understand a wide spectrum of questions and it would be interesting to investigate this further.

\section{CONCluding REMARKS}

At the end of his paper, Ramanujan [17] derives the expression

$$
\Lambda(n)=-\sum_{q=1}^{\infty} \frac{c_{n}(q)}{q},
$$

which is "dual" to the expansions we have considered till now. This suggests that perhaps there is a "dual theory" of such expansions of the form

$$
f(n)=\sum_{q=1}^{\infty} f^{*}(q) c_{n}(q) .
$$

Several extensions of Ramanujan's expansions of this type to generalizations of the von Mangoldt function appear in [13].

Finally, we mention the monograph of Sivaramakrishnan [20] which contains a wealth of material on Ramanujan sums including a curious "reciprocity law" which surely will find a place in a larger theory. We also highlight the relevance of the theory of almost periodic functions as studied in [14] where Ramanujan expansions are derived for certain strongly multiplicative function.

The interesting thing about the right hand side of (5) is that if the series converges absolutely and we replace $n$ by a real number $x$, we obtain a continuous function which interpolates the given arithmetical function. In this way, we can view the Ramanujan expansion as a continuous analogue of the discretely defined arithmetical function.

Acknowledgements. I would like to thank Kumar Murty, S. Gun, P. Rath, G. Gadiyar and R. Padma for their comments on an earlier version of this article.

\section{REFERENCES}

[1] T. Apostol, Arithmetical properties of generalized Ramanujan sums, Pacific Journal of Math., 41, No. 2, (1972), 281-293.

[2] R. Carmichael, Expansions of arithmetical functions in infinite series, Proc. London Math. Society, 34 (1932), 1-26. 
[3] H. Delange, On Ramanujan expansions of certain arithmetical functions, Acta Arithmetica, 31 (1976), 259-270.

[4] C. Fowler, S. R. Garcia, G. Karaali, Ramanujan sums as supercharacters, to appear in Ramanujan Journal.

[5] H.G. Gadiyar and R. Padma, Ramanujan-Fourier series, the Wiener-Khintchine formula and the distribution of prime pairs, Physica A., 269 (1999), 503-510.

[6] H.G. Gadiyar, M. Ram Murty and R. Padma, Ramanujan expansions and a theorem of Ingham, to appear in Indian Journal of Pure and Applied Mathematics.

[7] G.H. Hardy, Note on Ramanujan's trigonometrical function $c_{q}(n)$ and certain series of arithmetical functions, Proc. Cambridge Phil. Soc., 20 (1921), 263-271.

[8] G.H. Hardy and J.E. Littlewood, New proofs of the prime number theorem and similar theorems, Quarterly Journal of Math., 46 (1915), 215-219.

[9] G. H. Hardy and E.M. Wright, An introduction to the theory of numbers, 6th Edition, Oxford University Press, 2008.

[10] A. Hildebrand, Über die punktweise Konvergenz von Ramanujan-Entwicklungen zahlentheoretischer Funktionen, Acta Arithmetica, 44 (1984), no. 2, 109-140.

[11] A. Hildebrand, W. Schwarz and J. Spilker, Still another proof of Parseval's equation for almost even arithmetical functions, Aequationes Math., 35 (1988), no. 2-3, 132-139.

[12] O. Hölder, Zur Theorie der Kreisteilungsgleichung $K_{m}(x)=0$, Prace Matematyczno Fizyczne, 43 (1936), 13-23.

[13] A. Ivic, A property of Ramanujan's sums concerning totally multiplicative functions, Univ. Beograd. Publ. Elektrotehn. Fak., Ser. Mat. Fiz., 577-598 (1977), 74-78.

[14] M. Kac, E.R. van Kampen and A. Wintner, Ramanujan sums and almost periodic functions, American Journal of Math., 62 No. 1 (1940), 107-114.

[15] L.G. Lucht, A survey of Ramanujan expansions, International Journal of Number Theory, 6 (2010), 1785-1799.

[16] M. Ram Murty, Problems in analytic number theory, Springer, 2nd edition, 2008.

[17] S. Ramanujan, On certain trigonometric sums and their applications in the theory of numbers, Transactions of the Cambridge Phil. Society, 22 (1918), 179-199.

[18] W. Schwarz and J. Spilker, Arithmetical Functions, Lecture Note Series 184, London Mathematical Society, 1994.

[19] W. Schwarz, Ramanujan expansions of arithmetical functions, in Ramanujan Revisited, edited by G.E. Andrews, R.A. Askey, B.C. Berndt, K.G. Ramanathan, and R.A. Rankin, Academic Press, Boston, 1988, pp. 187-214.

[20] R. Sivaramakrishnan, Classical theory of arithmetic functions, Marcel Dekker, 1989.

[21] J. Spilker, Ramanujan expansions of bounded arithmetic functions, Archiv der Mathematik, 35 (1980), 451-453.

[22] N. Ushiroya, Mean value theorems for multiplicative arithmetic functions of several variables, Integers, 12 (2012), no. 5, 989-1002.

[23] A. Wintner, Eratosthenian averages, Waverly Press, 1943.

Department of Mathematics, Queen's University, Kingston, Ontario, K7L 3N6, CANADA

E-mail address: murty@mast.queensu.ca 